\title{
REVISÃO INTEGRATIVA DAS PROPRIEDADES PSICOMÉTRICAS DA STATE-TRAIT DEPRESSION SCALES
}

Gabriela da Silva Cremasco

Universidade São Francisco

Makilim Nunes Baptista

Universidade São Francisco
Recebido em: 08/04/2019

$1^{\text {a }}$ revisão em: 11/09/2019

Aceito em: 29/04/2020

\section{RESUMO}

O presente estudo apresenta uma revisão integrativa da literatura sobre as propriedades psicométricas da State-Trait Depression Scales. Foram consultadas as bases de dados Google Acadêmico e Periódicos CAPES e incluídos os artigos publicados entre os anos de 1995 e 2017. Dos 151 artigos, foram analisados 14 que corresponderam aos critérios de inclusão e exclusão. Os resultados indicaram que o instrumento possui propriedades psicométricas adequadas, podendo ser uma ferramenta útil na avaliação da depressão, inclusive para o contexto brasileiro. No entanto, são necessários estudos que busquem outros tipos de evidências de validade, com amostras mais amplas e de pacientes com diagnóstico de depressão.

Palavras-chave: depressão; testes psicológicos; propriedades psicométricas. 


\section{INTEGRATIVE REVIEW OF THE PSYCHOMETRIC PROPERTIES OF STATE-TRAIT DEPRESSION SCALES}

\section{ABSTRACT}

The present study presents an integrative review of the literature on the psychometric properties of State-Trait Depression Scales. The Google Academic and Periodical CAPES databases were consulted and articles published between 1995 and 2017 were included. Of the 151 articles, 14 were analyzed that corresponded to the inclusion and exclusion criteria. The results indicated that the instrument has adequate psychometric properties and may be a useful tool in the evaluation of depression, including in the Brazilian context. However, studies are needed that look for other types of evidence of validity, with larger samples and patients diagnosed with depression.

Keywords: depression; psychological tests; psychometric properties

\section{REVISIÓN INTEGRATIVA DE LAS PROPIEDADES PSICOMÉTRICAS DE LA STATE-TRAIT DEPRESSION SCALES}

\section{RESUMEN}

El presente estudio presenta una revisión integrativa de la literatura sobre las propiedades psicométricas de la State-Trait Depression Scales. Se consultaron las bases de datos Google Académico y Periódicos CAPES e incluidos los artículos publicados entre los años 1995 y 2017. De los 151 artículos, se analizaron 14 que correspondían a los criterios de inclusión y exclusión. Los resultados indicaron que el instrumento posee propiedades psicométricas adecuadas, pudiendo ser una herramienta útil en la evaluación de la depresión, incluso para el contexto brasileño. Sin embargo, son necesarios estudios que busquen otros tipos de evidencia de validez, con muestras más amplias y de pacientes con diagnóstico de depresión.

Palabras clave: depresión; pruebas psicológicas; propiedades psicométricas. 


\section{INTRODUÇÃO}

A depressão é classificada como a principal causa mental de incapacidade. Se caracteriza especialmente pela presença de humor deprimido e/ou anedonia, influenciando diretamente na qualidade de vida das pessoas acometidas. Trata-se de um problema de saúde pública, além de um dos principais fatores de risco para o suicídio (World Health Organization [WHO], 2017).

Para possibilitar um tratamento eficaz para cada pessoa, é necessário que a avaliação da depressão seja realizada adequadamente (Ely, Nunes, \& Carvalho, 2014). Nesse sentido, como indicado por Campos (2006), a depressão deve ser avaliada não só como um estado emocional, mas também como traço de personalidade, de modo a verificar questões referentes a estabilidade, cronicidade e frequência com que determinadas características são apresentadas.

Como proposto por Teasdale (1988), a natureza dos processos cognitivos negativos que se tornam ativos em um estado deprimido é que vai determinar a gravidade do quadro de depressão. Esses processos interagem com fatores como estressores ambientais, suporte social ou disposições biológicas. Nesse sentido, os sintomas da depressão são leves e transitórios em algumas pessoas, enquanto em outras são mais graves e persistentes. Uma das hipóteses para a recuperação mais rápida de algumas pessoas se refere a resposta do indivíduo frente aos sintomas apresentados.

Com objetivo de avaliar a depressão como traço e estado, Spielberger (1995) desenvolveu a State Trait Depression Scales (ST-DEP), sendo o estudo preliminar das propriedades psicométricas da escala publicado por Ritterband e Spielberger (1996). A escala avalia os aspetos cognitivos e afetivos da depressão, sendo dividido em duas subescalas (traço e estado). Conforme Spielberger e Reheiser (2009), a subescala de estado (S-DEP) mede a intensidade dos sentimentos e cognições depressivas em um determinado momento, sendo que indivíduos com escores altos na S-DEP tendem a terem experimentado sentimentos intensos de tristeza, por exemplo. Já a subescala de traço (T-DEP) mede a frequência com que as cognições e sentimentos relacionados a depressão são experimentados ao longo do tempo, sendo que pessoas com pontuações altas nessa subescala tendem a apresentar esses sentimentos mais frequentemente e de forma mais intensa, que podem se refletir em sintomas como desesperança, por exemplo.

A ST-DEP apresenta dois fatores para cada subescala intitulados de distimia (afeto negativo) e eutimia (afeto positivo). É composta por 20 itens em escala do tipo Likert de quatro pontos ( $1=$ "quase nunca"; 2 = "às vezes"; $3=$ "muitas vezes" e 4= "quase sempre"), sendo 10 para avaliação de estado (5 distimia e 5 eutimia) e 10 para traço ( 5 eutimia e 5 distimia), apresentando valores de confiabilidade acima de 0,80 . Portanto, avalia a intensidade da depressão em um determinado momento e enquanto características individuais de personalidade, bem como fornece medidas separadas de afetividade positiva e negativa associadas à 
depressão. Não inclui sintomas vegetativos (sono, apetite, fadiga) representando um diferencial em relação a outras escalas como o Inventário de Depressão de Beck (BDI), a Escala de Auto-Avaliação da Depressão de Zung (ZUNG) e a Center for Epidemiologic Studies (CES-D), que utilizam as pontuações desses sintomas em seus escores total, podendo acarretar em falsos positivos quando aplicados em pacientes com questões médicas associadas, devido a sobreposição de sintomas (Spielberger \& Reheiser, 2009).

Apesar de ser utilizada internacionalmente, a ST-DEP é pouco conhecida no Brasil, não existindo iniciativas de adaptação da escala para essa população. Por se tratar de um instrumento promissor para a avaliação da depressão, o objetivo do presente estudo foi verificar a produção científica internacional acerca das propriedades psicométricas da ST-DEP, por meio de uma revisão integrativa da literatura. Conforme relatado por Senra e Lourenço (2016), a revisão integrativa possibilita organizar e sintetizar os achados dos estudos sobre determinada temática, contribuindo para ampliar o conhecimento sobre o assunto em questão.

\section{MÉTODO}

\section{ESTRATÉGIA DE BUSCA}

A busca de artigos foi realizada nas bases de dados Google Acadêmico (https://scholar.google.com.br/) e Periódicos CAPES (http://www.periodicos.capes.gov.br/) no mês de setembro do ano de 2017. Em ambas as bases foram utilizados os seguintes termos de busca com operadores booleanos: ("State-Trait Depression Scales" OR "State-Trait Depression Questionnaire" OR "ST-DEP").

\section{CRITÉRIOS DE ELEGIBILIDADE}

Os critérios de inclusão empregados para a seleção das publicações foram: a) artigos publicados entre os anos de 1995 e 2017, sendo 1995 o ano em que os itens do instrumento foram construídos; b) estudos que tiveram como foco verificar as propriedades psicométricas da escala e c) estudos com amostras de adolescentes e adultos. Os critérios de exclusão aplicados foram: a) publicações em forma de dissertação, tese e trabalho de conclusão de curso; b) livros; c) resenhas; d) artigos teóricos e e) comentários.

\section{EXTRAÇÃO DOS DADOS}

Os estudos elegíveis foram avaliados inicialmente por meio da leitura dos títulos e dos resumos, sendo excluídos aqueles que não cumpriam os critérios de elegibilidade. Após a triagem inicial, os demais estudos foram lidos na íntegra, sendo novamente excluídos aqueles que não atingiam os critérios (ex: estudos com objetivo de verificar as propriedades psicométricas de outro instrumento que não a ST-DEP). Os artigos que compuseram a revisão foram avaliados conforme suas 
principais características, tais como, ano de publicação, periódicos, autoria, propriedade psicométrica avaliada, análises utilizadas e principais resultados obtidos em termos de propriedades psicométricas.

\section{RESULTADOS}

Foram obtidos um total de 151 publicações nas bases de dados (Google Acadêmico $=102$; Periódicos CAPES $=49$ ), sendo seis removidas por serem duplicadas. Além disso, foram recuperados dois estudos na íntegra e que não constavam nas bases de dados incluídas na pesquisa, mas que foram enviados a partir de contato por e-mail com um dos pesquisadores do instrumento, que cedeu os estudos. Após a leitura dos resumos, foram selecionados 24 trabalhos com base nos critérios de inclusão e exclusão. Os textos completos das publicações foram recuperados e, após a leitura na íntegra, foram excluídos mais 10 estudos, que tinham como objetivo verificar as propriedades psicométricas de um instrumento de avaliação de traço e estado de ansiedade, restando 14 artigos que foram incluídos na revisão, como mostra a Figura 1.

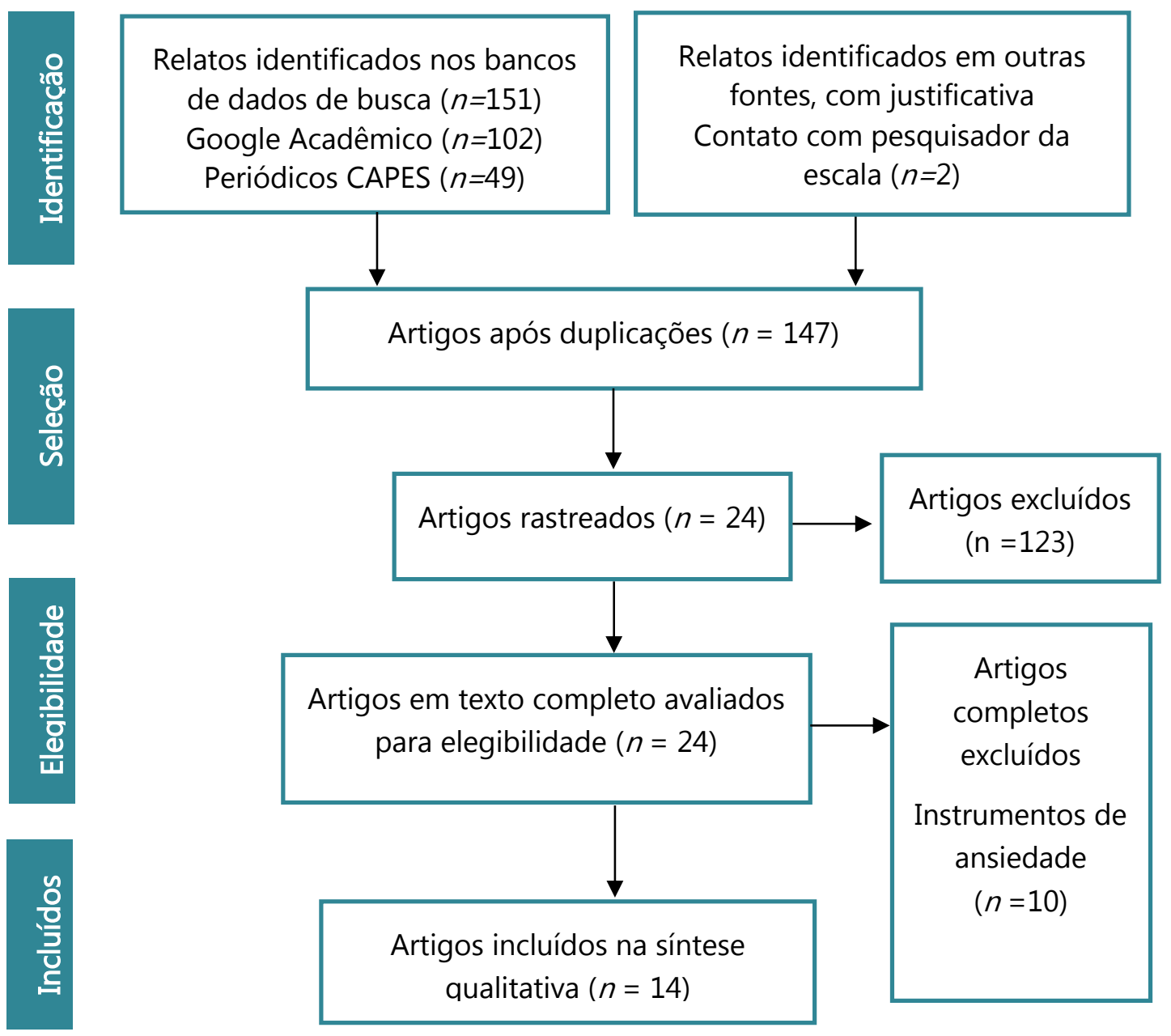

Figura 1. Diagrama de fluxo (adaptado de Moher, Liberati, Tetzlaff, Altman \& Prisma Group, 2009). 
Inicialmente, os artigos incluídos na revisão foram caracterizados quanto a autoria, ano de publicação e periódico. Esta descrição é apresentada na Tabela 1.

\section{Tabela 1.}

Autoria, ano e periódico.

\begin{tabular}{|c|c|c|c|}
\hline ID & Autoria & Ano & Periódico \\
\hline 1 & Ritterband \& Spielberger & 1996 & Depression and Stress \\
\hline 2 & Ritterband \& Spierlberger & 2001 & $\begin{array}{c}\text { Journal of Clinical Psychology } \\
\text { in Medical Settings }\end{array}$ \\
\hline 3 & Spaderna, Schmukle, \& Krohne & 2002 & Diagnostica \\
\hline 4 & $\begin{array}{l}\text { Spielberger, Carretero-Dios, } \\
\text { Santos-Roig, \& Buela-Casal }\end{array}$ & 2002 & $\begin{array}{c}\text { International Journal of Clinical } \\
\text { and Health Psychology }\end{array}$ \\
\hline 5 & $\begin{array}{l}\text { Spielberger, Carretero-Dios, } \\
\text { Santos-Roig, \& Buela-Casal }\end{array}$ & 2002 & $\begin{array}{c}\text { International Journal of Clinical } \\
\text { and Health Psychology }\end{array}$ \\
\hline 6 & $\begin{array}{c}\text { Krohne, Schmukle, Spaderna, \& } \\
\text { Spielberger }\end{array}$ & 2002 & Anxiey, Stress and Coping \\
\hline 7 & $\begin{array}{l}\text { Spielberger, Ritterband, } \\
\text { Reheiser, \& Brunner }\end{array}$ & 2003 & $\begin{array}{c}\text { International Journal of Clinical } \\
\text { and Health Psychology }\end{array}$ \\
\hline 8 & $\begin{array}{c}\text { Spielberger, Buela- } \\
\text { Casal, Agudelo, Carretero-Dios, } \\
\text { \& Santolaya }\end{array}$ & 2005 & Actas Espanolas de Psiquiatria \\
\hline 9 & Agudelo et al. & 2005 & Salud Mental \\
\hline 10 & Otálvaro & 2007 & Psicología desde el Caribe \\
\hline 11 & Vera-Villarroel et al. & 2008 & $\begin{array}{c}\text { International Journal of Clinical } \\
\text { and Health Psychology }\end{array}$ \\
\hline 12 & $\begin{array}{l}\text { Lehr, Hillert, Schmitz, \& } \\
\text { Sosnowsky }\end{array}$ & 2008 & Diagnostica \\
\hline 13 & Vera-Villarroel et al. & 2010 & Psychological Reports \\
\hline 14 & $\begin{array}{c}\text { Soto, Muños, \& Riaño- } \\
\text { Hernández }\end{array}$ & 2012 & $\begin{array}{c}\text { Revista Diversitas - } \\
\text { Perspectivas em Psicología }\end{array}$ \\
\hline
\end{tabular}

Em relação ao ano de publicação, foram obtidos estudos entre os anos de $1996 \mathrm{e}$ 2012 , sendo 2002 o ano com maior frequência de estudos ( $f=4 ; 28,57 \%$ ), seguido dos anos de 2005 e 2008 com duas publicações cada ( $f=2 ; 14,29 \%$ ). Dos 14 estudos, $(f=4 ; 28,57 \%)$ foram publicados no International Journal of Clinical and Health Psychology e ( $f=2 ; 14,29 \%)$ na Diagnostica. Charles Donald Spielberger, autor responsável pelo desenvolvimento do instrumento, fez parte da autoria de ( $f=10 ; 71,43 \%)$ publicações. 
A maior parte dos estudos teve amostra composta de estudantes universitários ( $f=10 ; 71,43 \%)$, seguido de adolescentes $(f=1 ; 7,14 \%)$, depressivos $(f=1 ; 7,14 \%)$, pacientes com câncer, saudáveis e psiquiátricos $(f=1 ; 7,14 \%)$ e adolescentes e universitários ( $f=1 ; 7,14 \%$ ). A caracterização com o tipo de amostra incluída em cada estudo será apresentada na Tabela 2. Com base na classificação de tamanho amostral de Prieto e Muñiz (2000), ( $f=10 ; 71,42 \%)$ estudos tiveram amostra moderada (entre 200 e 500 participantes), ( $f=1 ; 7,14 \%)$ pequena (inferior a 200 ) e $(f=3 ; 21,43 \%)$ amostra grande (mais de 500 ).

Tabela 2.

Caracterização das amostras dos estudos.

\begin{tabular}{ccc} 
ID & Amostra & $n$ \\
\hline 1 & Universitários & 251 \\
2 & Pacientes com câncer, saudáveis e & 188 \\
& psiquiátricos & \\
3 & Universitários & 611 \\
4 & Universitários & 264 \\
5 & Universitários & 264 \\
6 & Universitários & 1175 \\
7 & Universitários & 251 \\
8 & Universitários & 300 \\
9 & Universitários & 300 \\
10 & Adolescentes e universitários & 600 \\
11 & Universitários & 300 \\
12 & Depressivos & 450 \\
13 & Universitários & 301 \\
14 & Adolescentes & 314 \\
\hline
\end{tabular}

Em relação aos locais em que os estudos foram realizados, ( $f=4 ; 26,67 \%$ ) foram conduzidos na Espanha; ( $f=3 ; 20 \%$ ) na Alemanha e Estados Unidos; ( $f=2 ; 13,33 \%$ ) no Chile e $(f=1 ; 6,67 \%)$ no Canadá, Colômbia e Peru. Posteriormente, os estudos foram verificados a partir das propriedades psicométricas avaliadas, análises utilizadas e os principais resultados obtidos, como pode ser verificado na Tabela 3. Ressalta-se que a ordem dos artigos apresentadas pelo (ID) é a mesma em todas as tabelas. 
Tabela 3.

Caracterização dos resultados dos estudos avaliados.

\begin{tabular}{|c|c|c|c|c|}
\hline ID & $\begin{array}{c}\text { Propriedade } \\
\text { psicométrica } \\
\text { avaliada }\end{array}$ & Análises & $\alpha$ & Correlações \\
\hline 1 & $\begin{array}{c}\text { Estrutura } \\
\text { interna e } \\
\text { convergência }\end{array}$ & $\begin{array}{c}\text { AFE } \\
\text { Correlação de } \\
\text { Pearson }\end{array}$ & ST-DEP: 0,96 & $\begin{array}{l}\text { T-DEP e BDI: } 0,81 ; \text { T- } \\
\text { DEP e CES-D: } 0,83 ; \text { T- } \\
\text { DEP e ZUNG: } 0,88\end{array}$ \\
\hline 2 & Convergência & $\begin{array}{l}\text { Correlação de } \\
\text { Pearson }\end{array}$ & $\mathrm{N} / \mathrm{Q}$ & $\begin{array}{c}\text { S-DEP e BDI: } 0,76 ; \text { T-DEP } \\
\text { e BDI: } 0,69\end{array}$ \\
\hline 3 & $\begin{array}{c}\text { Estrutura } \\
\text { interna e } \\
\text { convergência }\end{array}$ & $\begin{array}{c}\text { AFE } \\
\begin{array}{l}\text { Correlação de } \\
\text { Pearson }\end{array}\end{array}$ & $\begin{array}{l}\text { S-DEP: 0,88 } \\
\text { T-DEP: 0,91 }\end{array}$ & $\begin{array}{l}\text { BDI e S-DEP: } 0,63 \\
\text { SDS e S-DEP: } 0,60 \\
\text { ADS e S-DEP: } 0,71 \\
\text { BDI e T-DEP: } 0,67 \\
\text { SDS e T-DEP: } 0,77 \\
\text { ADS e T-DEP: } 0,70\end{array}$ \\
\hline 4 & $\begin{array}{c}\text { Estrutura } \\
\text { interna e } \\
\text { convergência }\end{array}$ & $\begin{array}{c}\text { AFE } \\
\text { Correlação de } \\
\text { Pearson }\end{array}$ & T-DEP: 0,95 & $\begin{array}{l}\text { T-DEP e BDI: 0,78; T- } \\
\text { DEP e CBD: } 0,82 ; T- \\
\text { DEP e STAI-T: } 0,80\end{array}$ \\
\hline 5 & $\begin{array}{l}\text { Estrutura } \\
\text { interna e } \\
\text { convergência }\end{array}$ & $\begin{array}{c}\text { AFE } \\
\text { Correlação de } \\
\text { Pearson }\end{array}$ & S-DEP: 0,96 & $\begin{array}{c}\text { S-DEP e BDI: } 0,72 ; \text { S-DEP } \\
\text { e STAI: } 0,83\end{array}$ \\
\hline 6 & $\begin{array}{l}\text { Estrutura } \\
\text { interna e } \\
\text { convergência }\end{array}$ & $\begin{array}{c}\text { Correlação de } \\
\text { Pearson }\end{array}$ & $\mathrm{N} / \mathrm{Q}$ & $\begin{array}{l}\text { S-DEP e BDI: 0,58; S-DEP } \\
\text { e SDS: 0,66; S-DEP e CES- } \\
\text { D: 0,62; T-DEP e BDI: } \\
\text { 0,73; T-DEP e SDS: 0,82; } \\
\text { T-DEP e CES-D: } 0,79\end{array}$ \\
\hline 7 & $\begin{array}{c}\text { Estrutura } \\
\text { interna e } \\
\text { convergência }\end{array}$ & $\begin{array}{c}\text { AFE } \\
\text { Correlação de } \\
\text { Pearson }\end{array}$ & ST-DEP: 0,93 & $\begin{array}{l}\text { S-DEP e BDI: 0,57; S- } \\
\text { DEP e ZUNG: 0,66; T- } \\
\text { DEP e BDI: 0,76; S- } \\
\text { DEP e ZUNG: 0,85 }\end{array}$ \\
\hline 8 & Convergência & $\begin{array}{c}\text { Correlação de } \\
\text { Pearson }\end{array}$ & N/Q & $\begin{array}{l}\text { S-DEP e BDI: 0,59; S- } \\
\text { DEP e STAI-S: 0,70; T- } \\
\text { DEP e BDI: 0,74; T- } \\
\text { DEP e STAI-T: 0,52 }\end{array}$ \\
\hline 9 & $\begin{array}{l}\text { Estrutura } \\
\text { interna }\end{array}$ & AFE & $\begin{array}{c}\text { S-DEP (Distimia } \\
0,90 \text { e Eutimia } \\
0,92 \text { ) } \\
\text { T-DEP } \\
\text { (Distimia 0,88 } \\
\text { e Eutimia 0,91) }\end{array}$ & $\mathrm{N} / \mathrm{Q}$ \\
\hline
\end{tabular}


Tabela 3. Continuação.

\begin{tabular}{|c|c|c|c|c|}
\hline ID & $\begin{array}{c}\text { Propriedade } \\
\text { psicométrica } \\
\text { avaliada }\end{array}$ & Análises & $\alpha$ & Correlações \\
\hline 10 & $\begin{array}{c}\text { Estrutura } \\
\text { interna e } \\
\text { convergência }\end{array}$ & $\begin{array}{c}\text { AFE } \\
\text { Correlação de } \\
\text { Pearson }\end{array}$ & $\begin{array}{l}\text { S-DEP: } 0,82 \\
\text { T-DEP: } 0,83\end{array}$ & $\begin{array}{l}\text { T-DEP e BDI: 0,52; T- } \\
\text { DEP e STAI-T: 0,53; S- } \\
\text { DEP e BDI: 0,43; S- } \\
\text { DEP e STAI-S: } 0,67\end{array}$ \\
\hline 11 & $\begin{array}{c}\text { Estrutura } \\
\text { interna e } \\
\text { convergência }\end{array}$ & $\begin{array}{l}\text { AFE } \\
\text { Correlação de } \\
\text { Pearson }\end{array}$ & T-DEP: 0,90 & $\begin{array}{l}\text { T-DEP e BDI: } 0,64 ; \text { T- } \\
\text { DEP e SDS: } 0,71 ; \text { T- } \\
\text { DEP e STAI-T: 0,72 }\end{array}$ \\
\hline 12 & $\begin{array}{l}\text { Estrutura } \\
\text { interna }\end{array}$ & AFE & ST-DEP: 0,92 & N/Q \\
\hline 13 & $\begin{array}{c}\text { Estrutura } \\
\text { interna e } \\
\text { convergência }\end{array}$ & $\begin{array}{c}\text { AFE } \\
\text { Correlação de } \\
\text { Pearson }\end{array}$ & $\begin{array}{c}\text { S-DEP: 0,94 } \\
\text { (Distimia 0,96 } \\
\text { e Eutimia 0,97) }\end{array}$ & $\begin{array}{l}\text { S-DEP e STAI-S: 0,57; } \\
\text { S-DEP e BDI-R: 0,37 }\end{array}$ \\
\hline 14 & $\begin{array}{l}\text { Estrutura } \\
\text { interna }\end{array}$ & AFE & $\begin{array}{l}\text { T-DEP: 0,76 } \\
\text { S-DEP: } 0,68\end{array}$ & N/Q \\
\hline
\end{tabular}

Nota. N/Q: não se qualifica; Análise Fatorial Exploratória (AFE); Análise Fatorial Confirmatória (AFC); Comparative Fit Index (CFI); Standardized Root Mean Square Residual (SRMR); Chilean Experimental Version for Trait Depression Evaluation (T-DEP); Beck Depression Inventory (BDI); Zung Self-Rating Depression Scale (SDS); State-Trait Anxiety Inventory - Trait sub-scale (STAI-T); State-Trait Anxiety Inventory - State sub-scale (STAI-S); Spanish Experimental Version for Trait Depression Evaluation (TDEP); Basic Depression Questionnaire (CBD); Center for Epidemiologic Studies Depression Scale (CESD); General Depression Scale (ADS); Beck Depression Inventory Revised (BDI-R)

De forma geral, os estudos tiveram como objetivo buscar evidências de validade baseadas na estrutura interna e validade convergente para a ST-DEP. As principais análises conduzidas para atingir os objetivos propostos foram a AFE e a correlação de Pearson. Em todos os estudos de estrutura interna foram verificados dois fatores para cada subescala da ST-DEP (eutimia e distimia), assim como no estudo original, com valores de confiabilidade variando entre 0,68 e 0,96, sendo que alguns estudos apresentaram apenas os valores para cada subescala (S-DEP e T-DEP) e outros apenas o valor para o instrumento total.

Apenas o estudo de Lehr et al. (2008) apresentou, além da AFE, dados de sensibilidade e especificidade para a escala a partir da análise de Curva ROC (Receiver Operating Characteristic Curve) com uma amostra de pessoas com depressão. Já o estudo de Krohne et al. (2002) realizou, além da análise de correlação de Pearson, a Análise Fatorial Confirmatória (AFC), indicando os seguintes índices de ajuste para a S-DEP $(C F I=0,97 ; S R M R=0,04)$ e para a T-DEP $(C F I=0,97 ; S R M R=0,04)$. Os autores também testaram os índices de ajuste para a adaptação alemã do instrumento, sendo observados índices de ajuste similares a 
versão original da escala, tanto para a S-DEP $(C F I=0,96 ; S R M R=0,05)$, quanto para a T-DEP (CFI= 0,99; SRMR: 0,04).

Para a busca de evidências de validade convergente para a ST-DEP, foram utilizados alguns instrumentos de depressão, sendo o mais frequente nas pesquisas o $\mathrm{BDI}(f=10 ; 78,57 \%$ ). Foram obtidas correlações que variaram entre 0,52 e 0,81 para o BDI com a subescala de traço e entre 0,37 e 0,76 para o BDI com a subescala de estado.

\section{DISCUSSÃO}

O presente estudo teve como objetivo realizar um levantamento da produção científica internacional das pesquisas que verificaram as propriedades psicométricas da ST-DEP, a partir de uma revisão integrativa da literatura. Trata-se de um instrumento bastante utilizado, no entanto, são poucos os estudos que se propuseram a investigar suas características psicométricas.

O levantamento foi limitado a estudos a partir do ano de 1995, que corresponde ao ano em que o instrumento foi desenvolvido. Foram obtidos estudos entre 1996 e 2012 nas bases de dados e em contato com um dos autores que realizou os estudos iniciais com o instrumento. Entre os anos de 2013 e 2017 não foram encontrados estudos em termos de evidências de validade nas bases consultadas, o que pode ser decorrente do fato de o autor do instrumento e autor de $71,43 \%$ dos estudos encontrados ter falecido em 2013, o que pode explicar o decréscimo das investigações das propriedades psicométricas da escala.

Quanto as propriedades psicométricas investigadas, todos os estudos apresentaram boas evidências de validade e fidedignidade para a escala, demonstrando dois fatores para o instrumento. Um único estudo se propôs a realizar a AFC do instrumento, tanto na versão original da escala, quanto na sua adaptação alemã. Foram testados modelos de um e dois fatores, sendo obtido melhores índices de ajuste para o modelo de dois fatores (distimia e eutimia). Como proposto por Krohne et al. (2002), é necessário realizar novos estudos com a ST-DEP com intuito de verificar se a estrutura com dois fatores se mantém. É importante fazer algumas ressalvas. Os estudos foram realizados em sua maioria com amostras de estudantes universitários, sendo necessárias novas investigações com amostras de pacientes com depressão e comparar com amostras não clínicas de modo a verificar a adequação do instrumento para avaliar a depressão enquanto traço e estado.

Em termos de validade convergente, era esperado observar maiores correlações entre a subescala de estado de depressão com o BDI, pelo fato de ambos terem o mesmo objetivo. No entanto, as magnitudes das correlações obtidas com o BDI foram fortes com a subescala de traço de depressão e moderadas a fortes com a subescala de estado, conforme a classificação proposta por Cohen (1988). Resultados similares foram obtidos por Spielberger e Reheiser (2009) que também 
observaram magnitudes correlacionais mais fortes entre o BDI com a subescala de traço em comparação com a subescala de estado. Nesse sentido, os autores relataram que o BDI pode estar avaliando mais as diferenças individuais, ou seja, o traço de depressão, do que a sintomatologia depressiva em um espaço de tempo específico.

Uma outra hipótese para essas diferenças se refere as categorias sintomatológicas que compuseram cada instrumento de depressão que, apesar de avaliarem o mesmo construto, podem diferir substancialmente em termos de tipo de sintoma avaliado. Nesse sentido, Calil e Pires (1998) apresentaram as principais categorias de sintomas que podem basear a construção de um instrumento de depressão, tais como: humor, vegetativos ou somáticos, motores, sociais, cognitivos, ansiedade e irritabilidade. O BDI apresenta em sua composição itens relacionados a categoria afetiva (9,5\%), vegetativos (29\%), sociais (5\%), cognitivos, $(52 \%)$ e irritabilidade (5\%). Já a ST-DEP foi construída baseada apenas em categorias de humor e cognitivas da depressão. Portanto, para novos estudos é importante utilizar instrumentos construídos a partir de categorias de sintomas depressivos semelhantes para verificar a convergência entre essas medidas, além de outros instrumentos que avaliam o traço depressivo.

Ainda em relação às evidências de validade, a validade de conteúdo, por exemplo, não foi investigada em nenhum dos estudos. A validade de conteúdo pode ser verificada por meio da análise de juízes especialistas em relação a temática proposta com intuito de avaliar se os itens do instrumento constituem uma amostra representativa das características associadas a determinado construto (Pasquali, 2009).

\section{CONSIDERAÇÕES FINAIS}

Avaliar a depressão enquanto um traço de personalidade e como um estado emocional pode fornecer informações que irão nortear o tratamento de forma adequada (Campos, 2006). A presente revisão indicou que a ST-DEP pode ser uma ferramenta promissora, com parâmetros psicométricos adequados com amostras de contextos internacionais, podendo ser um diferencial para avaliação da depressão traço e estado. O instrumento não tem tradução para o Brasil, sendo uma sugestão para estudos futuros a verificação das propriedades psicométricas da ST-DEP para o contexto brasileiro, além de pesquisas com amostras mais amplas e de pacientes com depressão. Outro ponto relevante a ser destacado se refere ao fato de nenhum estudo revisado ter considerado estatísticas capazes de estimar os parâmetros de modelos, considerando as especificidades do traço e do estado em relação a estrutura latente. Conforme Andreasen, Grove e Maurer (1980) e Blashfield e Morey (1979) existe uma discussão sobre a depressão ser uma variável latente dimensional ou categórica. A perspectiva dimensional considera os diferentes níveis de comprometimento, ao passo que a categórica classifica a presença ou a ausência de alguma patologia, sendo desejável a realização de estudos que considerem ambas as perspectivas. 
Dentre as principais limitações da presente pesquisa, tem-se o número de bases consultadas, sendo importante ampliar a busca para futuras investigações sobre o instrumento objetivando recuperar mais publicações a respeito da escala, além das palavras-chave selecionadas, que poderiam ter sido mais amplas e não somente com o nome da escala.

\section{REFERÊNCIAS ${ }^{1}$}

*Agudelo, D., Carretero-Dios, H, Picabia, A. B., Pitti, C., Spielberger, C, \& Buela-Casal, G. (2005). Evaluación del componente afectivo de la depresión: Análisis factorial del ST/DEP revisado. Salud Mental, 28(3), 32-41.

Andreasen, N. C., Grove, W. M., \& Maurer, R. (1980). Cluster analysis and the classification of depression. The British Journal of Psychiatry, 137(3), 256-265. doi:10.1192/bjp.137.3.256

Blashfield, R. K., \& Morey, L. C. (1979). The classification of depression through cluster analysis. Comprehensive Psychiatry, 20(6), 516-527. doi:10.1016/s0010-440x(79)80004-8

Calil, H. M., \& Pires, M. L. N. (1998). Aspectos gerais das escalas de avaliação de depressão. Revista de Psiquiatria Clínica, 25(5), 240-244.

Campos, R. A. G. C. (2006). 'Depressivos somos nós': Um estudo de conceptualização e avaliação da personalidade depressiva e da depressão. (Tese de doutorado), Universidade de Évora, Évora, Portugal.

Cohen, J. (1988), Statistical power analysis for the behavioral sciences. Hillsdale, NJ: Erlbaum.

Ely, P., Nunes, M. F. O., \& Carvalho, L. F. (2014). Avaliação psicológica da depressão: Levantamento de testes expressivos e autorrelato no Brasil. Avaliação Psicológica, 13(3), 419-426.

*Krohne, H. W., Schmukle, S. C., Spaderna, H., \& Spielberger, C. D. (2002). The State-Trait Depression Scales: An international comparison. Anxiety, Stress and Coping, 15(2), 105-122. doi:10.1080/1061580029002842

*Lehr, D., Hillert, A., Schmitz, E., \& Sosnowsky, N. (2008). Screening depressiver Störungen mittels Allgemeiner Depressions-Skala (ADS-K) und State-Trait Depressions Scales (STDS-T) eine vergleichende evaluation von Cut-Off-Werten. Diagnostica, 54, 61-70. doi:10.1026/00121924.54.2.61

Moher, D., Liberati, A., Tetzlaff, J., Altman, D. G., \& Prisma Group. (2009). Preferred reporting items for systematic reviews and meta-analyses: The PRISMA statement. PLOS medicine, 6(7). doi: 10.1371/journal.pmed.1000097

*Otálvaro, L. E. O. (2007). Análisis correlacional del Cuestionario de Depresión Estado/Rasgo con una muestra de adolescentes y universitarios de la ciudad de Medellín (Colombia). Psicología desde el Caribe, 20, 28-49.

Pasquali, L. (2009). Psicometria. Revista da Escola de Enfermagem da USP, 43(SPE), 992-999. doi:10.1590/50080-62342009000500002

*Ritterband, L. M., \& Spielberger, C. D. (1996). Construct validity of the Beck Depression Inventory as a measure of state and trait depression in nonclinical populations. Depression and Stress, 2(2), 123-145.

*Ritterband, L. M., \& Spielberger, C. D. (2001). Depression in a cancer patient population. Journal of Clinical Psychology in Medical Settings, 8(2), 85-93. doi:10.1023/A:1009551809695

Senra, L. X., \& Lourenço, L. M. (2016). A importância da revisão sistemática na pesquisa científica. In M. N. Baptista, \& D. C. Campos (Eds.), Metodologias de Pesquisa em Ciências - Análise Quantitativa e Qualitativa (pp. 176-190). Rio de Janeiro, RJ: LTC.

*Soto, C. M., Muñoz, D. P., \& Riaño-Hernández, D. (2012). Análisis factorial exploratorio del Inventario de Depresión Estado-Rasgo (ST-DEP) en adolescentes. Revista Diversitas - Perspectivas en Psicología, 8(2), 319-330.

*Spaderna, H., Schmukle, S. C., \& Krohne, H. W. (2002). Bericht über die deutsche Adaptation der State-Trait Depression Scales (STDS). Diagnostica, 48, 80-89. doi:10.1026//0012-1924.48.2.80 Spielberger, C. D. (1995). State-Trait Depression Scales (Form X-1). Mind Garden, Palo Alto, CA.

*Spielberger C. D., Buela-Casal, G., Agudelo, D., Carretero-Dios, H., \& Santolaya, F. (2005). Analysis of convergent and discriminant validity of the Spanish experimental version of the State-Trait Depression Questionnaire (ST-DEP). Actas Espanolas de Psiquiatria, 33(6), 374-82. 
*Spielberger, C. D., Carretero Dios, H., Santos-Roig, M., \& Buela Casal, G. (2002). Spanish experimental version of the State-Trait Depression Questionnaire (ST-DEP): State sub-scale (S-DEP). International Journal of Clinical and Health Psychology, 2(1), 72-89.

*Spielberger, C. D., Carretero Dios, H., Santos-Roig, M., \& Buela Casal, G. (2002). Spanish experimental version of the State-Trait Depression Questionnaire (ST-DEP): Trait sub-scale (T-DEP). International Journal of Clinical and Health Psychology, 2(1), 51-69.

Spielberger, C. D., \& Reheiser, E. C. (2009). Assessment of Emotions: Anxiety, Anger, Depression and Curiosity. Applied Psychology: Health and Well-Being, 1(3), 271-302. doi:10.1111/j.17580854.2009.01017.x

*Spielberger, C. D., Ritterband, L. M., Reheiser, E. C., \& Brunner, T. M. (2002). The nature and measurement of depression. International Journal of Clinical and Health Psychology, 3(2), 209-234.

Prieto, G., \& Muñiz, J. (2000). Um modelo para evaluar la calidad de los test utilizados em España. Papeles del Psicólogo, 77, 65-72.

Teasdale, J. D. (1988). Cognitive Vulnerability to Persistent Depression, Cognition and Emotion, 2(3), 247-274. doi:10.1080/02699938808410927

*Vera-Villarroel, P., Buela-Casal, G., Celis-Atenas, K., Córdova-Rubio, N., Encina-Olea, N., \& Spielberger, C. D. (2008). Chilean experimental version of the State-Trait Depression Questionnaire (ST-DEP): Trait sub-scale (T-DEP). International Journal of Clinical and Health Psychology, 8(2), 563-575. doi:10.2466/PR0.106.1.65-77

*Vera-Villarroel, P., Buela-Casal, G., Zych, I. Córdova-Rubio, N. Celis-Atenas, K., Zepeda, L., \& Spielberger, C. D. (2010). Chilean experimental version of the State-Trait Depression Questionnaire (ST-DEP): State subscale (S-DEP). Psychological Reports, 106(1), 65-77. doi:10.2466/PR0.106.1.65-77

World Health Organization (WHO) (2017). Depression and other common mental disorders: Global health estimates.

\section{CONFLITOS DE INTERESSES}

Não há conflitos de interesses.

\section{FINANCIAMENTO}

Coordenação de Aperfeiçoamento de Pessoal de Nível Superior - CAPES.

\section{SOBRE OS AUTORES}

Gabriela da Silva Cremasco é psicóloga pela Universidade São Francisco (2015). Mestre (2017) e Doutoranda pelo Programa de Pós-Graduação Stricto Sensu em Psicologia com ênfase em Avaliação Psicológica pela Universidade São Francisco como Bolsista pela Coordenação de Aperfeiçoamento de Pessoal de Nível Superior (CAPES).

E-mail: gabisilva10@hotmail.com

(1) https://orcid.org/0000-0003-2075-8049

Makilim Nunes Baptista é psicólogo. Doutor pelo Departamento de Psiquiatria e Psicologia Médica pela Universidade Federal de São Paulo. Professor do Programa de Pós-Graduação Stricto Sensu em Psicologia da Universidade São Francisco e Bolsista Produtividade pelo Conselho Nacional de Desenvolvimento Científico e Tecnológico.

E-mail: makilim01@gmail.com

(1) https://orcid.org/0000-0001-6519-254X

\footnotetext{
${ }^{1}$ Referências marcadas com o símbolo * fizeram parte da revisão sistemática.
} 